\title{
EMBOLISM AFTER PENICILLIN-OIL-BEESWAX
}

\author{
BY
}

\author{
A. W. FRANKLAND \\ Wright-Fleming Institute of Microbiology, St. Mary's Hospital, London
}

(RECEIVED FOR PUBLICATION, MAY 19, 1948)

The occurrence of fat embolism after injury to bone has been described and the literature adequately reviewed by Robb-Smith (1941) and by Rowlands and Wakeley (1941). Similar findings occur after the accidental intravenous introduction of oily substances. Grossmann (1946) described the $x$-ray appearances of two cases of pulmonary oil embolism following salpingography. The first patient had no symptoms for two days; the second patient began to cough very soon after the injection of the oil. The $x$-ray films in the latter patient were taken within five minutes, when it was seen that the main filling of the pulmonary arterial tree occurred in the lower lobes. Many observers have stressed the delayed appearance of symptoms which occur after fat embolism. Harris and others (1939) suggested that this is due to hydrolysis of fat particles with the release of free fatty acids. Animal experiments that were carried out suggested that severe reactions may occur after an oil-beeswax mixture, not only because the mixture blocks large branches of the pulmonary artery, but also because of the severe inflammatory reaction elicited by beeswax.

When a patient dies the recognition of a fat or oil embolism is not easy, because special methods have to be used to show the presence of these substances in the sections. In patients who recover the clinical picture is not clearly defined or easily recognized because the diagnosis has not been considered, especially where the symptoms are delayed. The development of pulmonary and cerebral signs and symptoms with petechial haemorrhages in the skin should all be looked for. Fat globules in the sputum and/or urine confirm the diagnosis. In many cases no abnormal signs are present and the symptoms are minimal.

I can find only one previous published account of oil embolism following penicillin in oil-wax therapy. Bondy and others (1947) describe a patient who had a severe reaction which showed maximal radiological changes on the second and third day after injection. The immediate effects ${ }_{\odot}^{+}$ were slight, consisting only of a penicillin-like taste in the mouth and a few coughs.

Two cases that occurred over two years ago had symptoms corresponding to a third describede below, in that these symptoms were immediate and? minimal, consisting of a tight feeling in the chesto and a cough immediately after receiving an injec-o tion of penicillin in oil-wax.

\section{Case Report}

A married woman, aged 30 , had been admitted for streptomycin therapy for persisting ulcerative colitis $\mathbb{D}$ While in the ward she had complained of chronic bronchitis, and by the time she had finished the streptomycin therapy there was no doubt that she had developed true asthmatic attacks. While await ing discharge from hospital she was given an injec? tion of 600,000 units in $2 \mathrm{ml}$. of calcium penicillin The penicillin was suspended in peanut oil with $4 . \overline{8}$ per cent $(w / v)$ beeswax B.P. The injection was giveng by a nurse who, on being questioned, could not be certain that the usual precautions of making sure thap the needle was not in a vein had been carried out? Immediately after the injection the patient began to cough, said she felt sick, asked for a bed-pan, and then said she was going to faint. She was put back to bed and when seen five minutes later she said she was feeling quite well. She was surprised the injecor tion had made her feel faint, as previously she had had many injections of either penicillin or streptos mycin without ill effect. When she was examinedu there were no abnormal physical signs, other tha those due to generalized bronchitis which had beex noted previously. The bronchitis was productive of a little sputum, about $20 \mathrm{ml}$. of which was collected? during the following day: this amount represented the expectoration from the time of waking until befor $\bar{B}$ the midday meal. Unfortunately no precautions wero taken to make sure that this was not contaminated with any oil or fat from a food source. A furtheo specimen of sputum was collected three days later and a control specimen of sputun taken from another 
chronic bronchitic patient. Sudan III was used as an oil stain. Only in the specimen collected the first day after the penicillin injection and only in certain parts of the field could many oil globules be seen; all these were extracellular, and most ${ }^{\prime}$ of them were very minute-about $5 \mu$ in diameter-though a few of the large ones were $50 \mu$ in size. Examination of a slide with the stained sputum on it, and a control of the unstained and also stained penicillin in oil-wax, showed that in no case was there rotation of the plane of polarized light. It is therefore suggested that the oil in the sputum had as its source the penicillin oil-wax injection given into the buttock.

\section{Summary}

A case is described in which a mild immediate reaction occurred after an injection of penicillin in oil-wax. It would appear that this was due to oil embolism.

\section{REFERENCES}

Bondy, P. K., Sheldon, W. H., and Weens, H. S. (1947). Amer. J. Med., 11i, 34

Grossmann, M. E. (1946). Brit. J. Radiol, 19, 178

Harris, R. I., Perrett, T. S., and MacLachlin, A. (1939). Ann. Surg., $110,1095$.

Robb-Smith, A. H. T. (1941). Eancet, 1, 135

Rowlands, R. A., and Wakeley, C. P. G. (1941). Lancet, 1, 502. 